\title{
Spectrophotometric Methods for Quantifying Pigmentation in Human Hair-Influence of MC1R Genotype and Environment
}

\author{
Sri N. Shekar ${ }^{\star 1,2}$, David L. Duffy ${ }^{1}$, Tony Frudakis ${ }^{3}$, Grant W. Montgomery ${ }^{1}$, Michael R. James ${ }^{1}$, \\ Richard A. Sturm ${ }^{4}$ and Nicholas G. Martin ${ }^{1}$ \\ ${ }^{1}$ Genetic Epidemiology Unit, Queensland Institute of Medical Research, Brisbane, Qld, Australia \\ ${ }^{2}$ School of Medicine (Central), University of Queensland, Brisbane, Qld, Australia \\ ${ }^{3}$ DNAPrint, Genomics, Inc., Sarasota, FL \\ ${ }^{4}$ Institute for Molecular Bioscience, University of Queensland, Brisbane, Qld, Australia \\ Received 4 June 2007, accepted 3 October 2007, DOI: 10.1111/j.1751-1097.2007.00237.x
}

\begin{abstract}
Eumelanin (brown/black melanin) and pheomelanin (red/yellow melanin) in human hair can be quantified using chemical methods or approximated using spectrophotometric methods. Chemical methods consume greater resources, making them less attractive for epidemiological studies. This investigation sought to identify the spectrophotometric measures that best explain the light-dark continuum of hair color and the measure that is best able to distinguish red hair from nonred hair. Genetic analysis was performed on these two measures to determine the proportion of genetic and environmental influences on variation in these traits. Reflectance curves along the visible spectrum and subjective ratings of hair color were collected from 1730 adolescent twin individuals. Discriminant class analyses were performed to determine the spectrophotometric measure that could best proxy for eumelanin and pheomelanin quantities. The ratio of light reflected in the green portion of the spectrum to that reflected in the red portion of the spectrum was best able to distinguish red hair from nonred hair. Melanocortin 1 receptor (MC1R) genotype explained some, but not all, variation in this measure. Light absorbed in the red portion of the spectrum was best able to explain the light-dark continuum of hair color. Variance components analysis showed that there were qualitatively different genetic influences between males and females for the light-dark continuum of hair. Our results show that spectrophotometric measures approximating variation in eumelanin and pheomelanin may be considered as an alternative to chemical methods in larger epidemiological studies.
\end{abstract}

\section{INTRODUCTION}

While variation in skin pigment shows marked differences between the major ethnic groups, most of the variation in hair pigment is within European populations (1). The development of chemical methods of quantifying eumelanin and pheomelanin in human hairs has been reviewed by Wakamatsu and Ito (2). Of the melanin in black and brown hairs, over $95 \%$ is eumelanin and the rest pheomelanin. Blond and red hairs have less melanin in total than black/brown hairs. Blond hairs have

*Corresponding author email: sri.shekar@qimr.edu.au (Sri N. Shekar) (c) 2007 The Authors. Journal Compilation. The American Society of Photobiology 0031-8655/08 a similar ratio of eumelanin to pheomelanin as that of dark brown hairs, while red hairs have approximately $67 \%$ eumelanin and 33\% pheomelanin (3). Biochemical studies have succeeded in identifying many of the genes and pathways of variation in skin and hair color (reviewed by Sturm [4]).

The melanocortin 1 receptor (MC1R) is a G-protein coupled receptor primarily expressed in melanocytes. Stimulation by $\alpha$-melanocyte-stimulating hormone, a natural agonist, leads to an elevated ratio of eumelanin over pheomelanin (5). Some alleles of the MC1R gene are associated with red hair, fair skin (6) and increased cancer risk (7). This set of traits, commonly called the red hair color (RHC) phenotype, generally shows recessive inheritance. Box et al. (8) showed that MC1R genotype was necessary but not sufficient to explain red hair.

A number of studies have examined correlations between eumelanin and pheomelanin levels and various spectrophotometric measures. Most of these have concentrated on skin reflectance, showing that eumelanin absorbs light across the visible spectrum with the greatest power around $650 \mathrm{~nm}$ ([9]; see also Edwards and Duntley [10]). A handful of studies have validated spectrophotometric measures of melanin in human hairs. Ozeki et al. (11) found that absorbance at $500 \mathrm{~nm}$ correlated highly with the quantity of chemically determined melanin (eumelanin and pheomelanin) while Ozeki et al. (12) found that the ratio of absorbance at $650 \mathrm{~nm}$ to $500 \mathrm{~nm}$ was able to explain the ratio between eumelanin to total melanin. This implies that absorbance at $650 \mathrm{~nm}$ is proportional to eumelanin content.

Spectrophotometric methods of quantifying pheomelanin were first explored by Gardner and MacAdam (13). They noticed an upward deviation of the reflectance curve of red headed individuals between 520 and $550 \mathrm{~nm}$. To capture this change, Reed (14) calculated the slope between the reflectance values at 400 and $550 \mathrm{~nm}$, accounting for the reflectance at $650 \mathrm{~nm}$ (known as the $R$ index). The $R$ index was subsequently used by Little and Wolff (15) to show that red headed women have little eumelanin in their hair.

In this study, we develop new estimates of eumelanin and pheomelanin. We validate these by examining the correlation between our measures and existing measures, to MC1R genotype, and by observing the correlation of the different measures between family members. 


\section{MATERIALS AND METHODS}

Samples. The data for the adolescent cohort used in this study were collected as part of a longitudinal study investigating the development of melanoma risk factors, particularly melanocytic naevi (moles). Twins were enlisted through contacting principals of primary schools in the greater Brisbane area, through word of mouth and a range of media. The twins who registered their interest were contacted and participation was conditional upon the informed consent of the twins and their parents. Details of the clinical protocol are described in McGregor et al. (16) and Zhu et al. (17). Approval to undertake this study was granted by the Human Research Ethics Committee of the Queensland Institute of Medical Research.

Hair reflectance spectrophotometry. Twins and their siblings were taken through the protocol when the twins were approximately 12 and 14 years of age. At each visit, the nurse classified each participant's hair into one of the five categories: fair/blonde, light brown, red/auburn, dark brown or black. In addition, a sample of hair between 2 and $5 \mathrm{~cm}$ was cut from the nape of the neck. For individuals with short hair (predominantly males), a sample was taken from the crown. A sample was not taken in the case where the hair was too short or dyed. For each sample, the intensity of light reflected between 340 and $1026 \mathrm{~nm}$ was captured using a UV-visible wavelength Ocean Optics USB2000 spectrophotometer (Ocean Optics, Inc., Dunedin, FL). Hair samples were placed before a brown background. The reading was taken at such an angle, and the resealable plastic collection bag stretched such that there was little reflectance of light from the bag itself. This was confirmed when there was found to be no significant difference in reflectance across hair colors when the process was repeated without the plastic bag. As the samples did not have to be removed from, and replaced into, the bag, considerable time was saved and the possibility of contamination between samples eliminated. Reflectance was captured for each hair sample at areas observed to have distinct colors, resulting in between one and six reflectance curves per individual. Reflectance values across the samples taken within each individual were averaged to obtain one reflectance curve for each individual. There were 1730 individuals for whom hair color was categorized and reflectance curves were recorded.

$M C 1 R$ genotype. Venous blood samples were collected from twins and siblings into EDTA tubes. DNA was extracted from buffy coats subsequent to salting out cellular proteins (18). The procedure for determining MC1R genotype is outlined elsewhere (19). Additional genotypes were obtained using a Compact MALDI-TOF Mass Spectrometer (Sequenom) (20), through an optimized multiplex assay of all common and a subset of rare known variants of MC1R. Variant alleles of MC1R strongly associated with the RHC phenotype, namely D84E, R151C, R160W and D294H, are denoted ' $R$ '. Individuals denoted ' $R / R$ ' are homozygous for one of these variant alleles. Individuals denoted as wildtype have wildtype alleles at the aforementioned loci in addition to having the wildtype allele at the V60L, V92M, R142H, I155T and R163Q loci.

Evaluating spectrophotometric measures of eumelanin and pheomelanin. To determine which spectrophotometric measures best approximate pheomelanin and eumelanin content in hair color, analyses were conducted to see how well the variance of each measure could be used to discriminate between classifications of observed hair color (discriminant class analysis using SPSS release 13.0; SPSS, Inc., Chicago, IL). Aside from existing spectrophotometric measures of pheomelanin and eumelanin, alternative measures were postulated to investigate further hypotheses. Spectrophotometric approximations of eumelanin were compared to see which could best explain the ordered categories fair/blonde, light brown, dark brown and black. Spectrophotometric approximations of pheomelanin were compared to see which could best distinguish individuals classified as red from those in other categories.

Zygosity testing. Zygosity of the twin pairs was established by typing them using the ABI Profiler Plus ${ }^{\mathrm{TM}}$ marker set and subsequently confirmed by typing many more markers for linkage analysis.

Testing of mean and variance homogeneity. Tests were performed on the indices to best explain light-dark (blonde-black) and red hair to determine whether means and variances were homogeneous across the sample independent of birth order, zygosity and sex $(21,22)$. Twice the difference in $\log$ likelihoods between the full and submodels is distributed as chi-squared with the degrees of freedom equal to the difference in degrees of freedom between the two models (likelihood ratio test) (23). Subsequent variance components models were based on the most parsimonious model to explain the data for each variable.

Twin pair covariance heterogeneity and variance components modeling. Twin pair covariances were used to decompose interindividual variation into genetic and environmental components. If the covariance between monozygotic (identical) twin pairs is greater than that of dizygotic (nonidentical) twin pairs, we assume that familial aggregation is driven by genetic influences, as monozygotic twin pairs share all their genes while dizygotic twin pairs share roughly half their genes. If partly different genes influence the trait in males and females (nonscalar sex limitation) this results in a lower correlation between brother and sister (opposite sex) twin pairs than would be predicted from the brother-brother and sister-sister (same sex) twin correlations (24).

Genetic modeling. Using the known basis for similarity of monozygotic and dizygotic twins, twin pair correlations can be used to decompose the variance of a trait into genetic and environmental influences. Genetic variation can be subdivided into additive (A) and nonadditive (D) influences. Additive genetic influences are those where the effect on the trait is the sum of the effects of the alleles influencing the trait. Monozygotic twin pairs are perfectly correlated for additive effects while dizygotic twin pairs, who share roughly half their genes, are expected to correlate about 0.5 . Nonadditive influences can be allelic interactions (dominance) or nonallelic interactions (epistasis). There is no possibility of distinguishing between dominance and epistasis in a classical twin design even though the nonadditive term is usually called "dominance," with dizygotic twins expected to correlate 0.25 . However, a significant estimate of D may suggest that either dominance, epistasis, or both, are significant contributors to variation.

Variation resulting from the environment can be modeled as either that influencing both twins (C) or that influencing each twin uniquely (E). If familial aggregation for a trait is influenced purely by a common environment $(\mathrm{C})$, we expect the twin pair correlation for monozygotic twins to be the same as that for dizygotic twins. By definition, twins are not correlated for unique environmental effects (see refs. 23-25 for further details on genetic modeling).

Based on inspection of the monozygotic and dizygotic correlations, either an ACE or an ADE model was fitted to each variable using the computer program Mx (1.61e) (26). Nested models were fitted by dropping $\mathrm{A}, \mathrm{C}$ and $\mathrm{D}$ in appropriate combinations. The unique environmental parameter, E, includes measurement error so it cannot be removed from the model. The likelihood ratio test was used to assess the fit of submodels (23).

If opposite sex twin pair covariance was significantly lower than dizygotic same sex twin pair covariances, indicating that a trait was nonscalar sex-limited, a general sex-limitation model that allows for quantitative differences in the sources of variation (common effects) between sexes, as well as qualitative differences in genetic influences between males and females, was fitted to the data (23). The method of modeling gender differences using twins and their siblings in $\mathrm{Mx}$ is outlined in Medland (27).

\section{RESULTS}

Rather than look at the multitude of wavelengths independently, we consider three areas of the reflectance curve as they relate to the three colors of light perceived by human rhodopsin-blue (below ca $490 \mathrm{~nm}$ ), green (between 490 and ca $560 \mathrm{~nm}$ ) and red (from ca $610 \mathrm{~nm}$ upwards). The averaged reflectance values of individuals within each of the five categories are shown in Fig. 1. Eumelanin content, observed as progressively darker hair color on a blonde-black continuum (3), appears to be inversely proportional to reflectance values in the green and red portions of the spectrum. As stated by Borges et al. (3), hair that is perceived as red contains a greater proportion of pheomelanin than brown/black hair while those with brown hair colors have greater eumelanin 


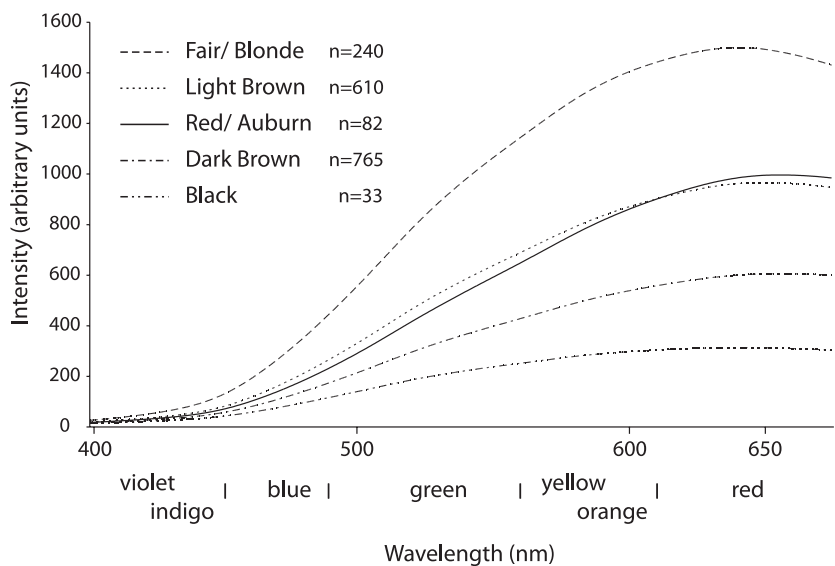

Figure 1. Reflectance curves averaged for individuals in each of the five categories of hair color collected.

than those with red or blonde hair. Cursory examination of the average curve for those classified as having red hair shows that red hair appears to absorb more light in the green portion of the spectrum and reflect more light in the red portion of the spectrum compared to the average reflectance of those classified as having light brown hair (Fig. 1).

The $R$ index was calculated according to the equation proposed by Reed (14):

$$
R=\frac{100\left(y_{530}-0.243 y_{400}\right)}{y_{650}}
$$

where $y_{x}$ is the reflectance of light at wavelength $x$. This measure captures light absorbed in the green portion of the spectrum while accounting for eumelanin content using reflectance at $650 \mathrm{~nm}$. To investigate the possibility of pheomelanin reflecting light in the red portion of the spectrum in addition to absorbing light in the green portion of the spectrum, we postulate an alternative measure of pheomelanin. To account for eumelanin in the alternative model, a wavelength is required that is not influenced by pheomelanin (i.e. neither red nor green). We suggest $600 \mathrm{~nm}$ as a wavelength approximately equidistant between the red and green portions of the visible spectrum as a measure that should account for eumelanin in the alternative model. So, to quantify the effect of pheomelanin on the green and red portions of the spectrum, we consider the difference in reflectance intensity between these two colors, accounting for eumelanin content using $600 \mathrm{~nm}$ :

$$
P=\frac{y_{650}-y_{530}}{y_{600}}
$$

(pheomelanin index-herein called the $P$ index).

To determine which of these two indices $(R$ or $P)$ are best able to distinguish red hair from other categories, discriminant class analyses were performed. If the $P$ index is better able to distinguish red hair from nonred hair, then it is more probable that pheomelanin reflects light in the red portion of the spectrum in addition to absorbing light in the green portion of the spectrum. Correlations between the expected categorization from the discriminant class analysis and the observed categorization showed the $R$ index of Reed (14) to be a better predictor of red hair, although not significantly so (Table 1). To further confirm that pheomelanin absorbs light in the green
Table 1. Tetrachoric correlation of observed red $v s$ nonred hair categorization with that expected from a discriminant class analysis of the $R$ and $P$ indices of pheomelanin content, respectively.

\begin{tabular}{lccc}
\hline & Correlation & Lower CI & Upper CI \\
\hline$R$ index & 0.74 & 0.55 & 0.86 \\
$P$ index & 0.69 & 0.52 & 0.82 \\
\hline
\end{tabular}

portion of the spectrum, reflectance averaged across individuals with wildtype MC1R alleles was plotted alongside reflectance averaged across individuals homozygous for the high penetrance MC1R red hair alleles D84E, R151C, R160W and D294H (denoted R/R; [19]), as shown in Fig. 2. Each curve was standardized to the averaged reflectance at $650 \mathrm{~nm}$ to account for eumelanin content.

Considering this result, it is expected that reflectance or absorbance in the red portion of the spectrum captures variation in eumelanin content without being confounded by pheomelanin content. Three possible spectrophotometric approximations of eumelanin are considered here: absorbance at $650 \mathrm{~nm}(\mathrm{~A} 650 ; 12)$, reflectance at $600 \mathrm{~nm}(\mathrm{R} 600)$ and the widely used melanin index (28). Again, the polychoric correlation between the observed categorization of hair color and the categorization expected from the discriminant class analyses were used to determine the measure that best explained hair color. There was no significant difference between the ability of A650 ( $r=0.85)$ and $\mathrm{R} 600(r=0.86)$ to distinguish between different hair colors (Table 2). The melanin index $(r=0.79)$ was significantly less able to distinguish between different hair color categories than the other two measures. This reduction in power may be due to the logarithmic transformation of reflectances averaged around $655 \mathrm{~nm}$. However, the melanin index is more normally distributed than either of the other two indices.

In a spectrophotometric analysis, individuals with only pheomelanin in their hair will still reflect light in the red portion of the curve, confounding a spectrophotometric approximation of eumelanin. One way of circumventing this is to remove those with red hair in spectrophotometric analyses involving eumelanin. It is possible to identify these individuals through visual inspection or, to some extent, through MC1R

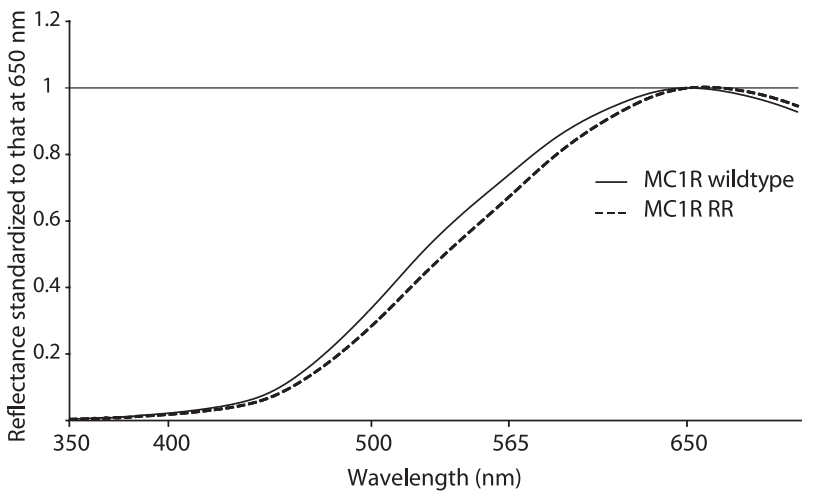

Figure 2. Averaged reflectance curves of those with two MC1R wildtype alleles $(n=186)$ and those with two MC1R variant alleles $(n=33)$, standardized to reflectance at $650 \mathrm{~nm}$. Although not shown for clarity, the reflectance curve for MC1R heterozygotes closely follows that of the MC1R wildtype homozygotes. 
Table 2. Polychoric correlation of observed hair color on a four point scale with expected categorization based on a discriminant analysis of three indices of eumelanin content.

\begin{tabular}{lccc}
\hline & Correlation & Lower CI & Upper CI \\
\hline Melanin index & 0.79 & 0.77 & 0.80 \\
Absorbance at $650 \mathrm{~nm}$ & 0.85 & 0.83 & 0.86 \\
Reflectance at $600 \mathrm{~nm}$ & 0.86 & 0.85 & 0.87 \\
& & & \\
\hline
\end{tabular}

genotype (8). It does not appear that the $R$ index can be used to distinguish red headed individuals from those with other colors. Figure 3 shows that the distribution of the $R$ index for individuals classified with red hair overlaps significantly with the distribution of the $R$ index for individuals classified as having light and dark brown hair. There is a significant difference in mean $R$ index values between those homozygous for the wildtype allele at all MC1R variant loci compared to those homozygous for the high penetrance MC1R red hair alleles $(P<0.00001)$, although the distributions of the $R$ index for these groups also overlap (Fig. 4). A receiver operating characteristic curve (Fig. 5) shows that, in our sample of individuals genotyped at MC1R, all three individuals with an $R$ index value below 39.9, and five of eight individuals with an $R$ index value below 43, were homozygous for the highly penetrant red hair alleles $(\mathrm{R} / \mathrm{R})$. However, across the sample, only $72 \%$ with an $R$ index value below 39.9 are classified as having red hair, although this may be caused by disparity of the number of individuals between categories. Of those classified as having red hair, only $16 \%$ have an $R$ index value below 39.9. Those individuals classified as having red hair $(4.6 \%$ of sample) were removed from the subsequent genetic analysis of the A650 index.

The distribution of absorbance at $650 \mathrm{~nm}$ was moderately negatively skewed and was transformed by reflecting the measure and taking the logarithm, resulting in the A650t

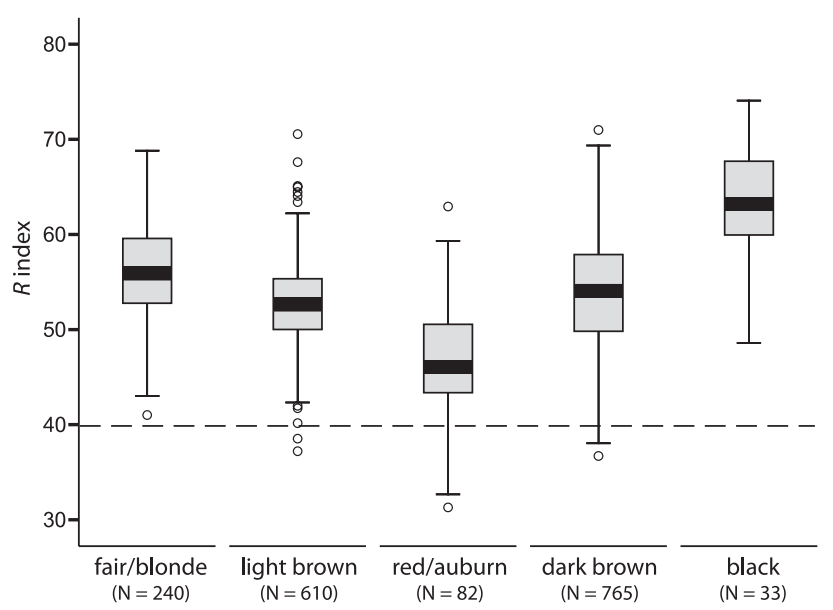

Figure 3. Red/auburn hair cannot be completely distinguished from other hair colors using the $R$ index. Boxes indicate lower quartile, median and upper quartile for the $R$ index within each rated category of hair color. Whiskers extend to the smallest and largest observation within 1.5 times the interquartile range below and above the lower and upper quartiles, respectively. Outliers greater than 1.5 times the interquartile range are indicated.

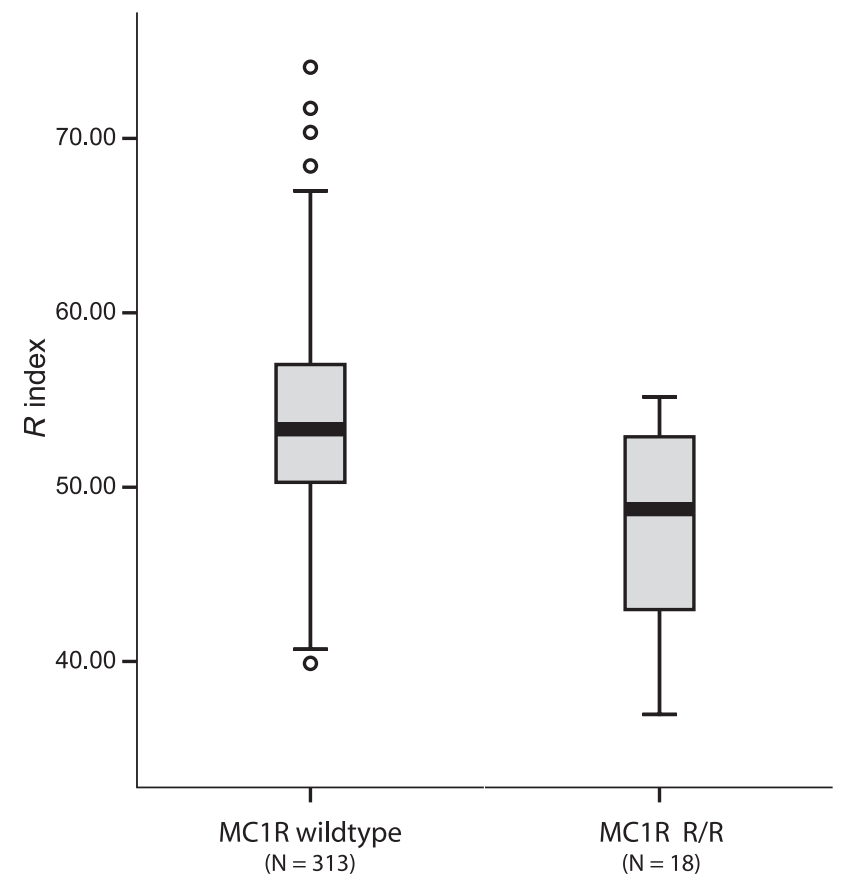

Figure 4. MC1R genotype does not completely explain red hair as captured by the $R$ index. MC1R R/R are those individuals who are homozygous for the variant allele at the highly penetrant red hair color alleles D84E, R151C, R160W or D294H on the MC1R gene.

measure. The A650t measure was used for further genetic analysis.

\section{Reliability}

To determine the reliability of the nurse ratings of hair color, 82 individuals were rated again $c a 6$ months after the first visit. The polychoric correlation between the hair color ratings between the two measurements was 0.966 (SE 0.012). For the spectrophotometric measurement of hair color, the samples for four individuals with different hair color ratings were remeasured five times. The intraclass correlation for the reflectance at $650 \mathrm{~nm}$ was $0.989(0.955,0.999)$; the intraclass correlation for the transformed A650 index (A650t) was 0.814 (0.480 $0.985)$. However, the polyserial correlation between the former and ordered ratings for hair color is lower $(-0.73)$ than that for the latter $(-0.775)$ using the same number of individuals $(n=1618)$. This correlation, -0.775 , is the same as the polyserial correlation between the melanin index and ordered ratings of hair color in this sample.

$R$ index values were calculated for the same four individuals for whom spectrophotometric curves were remeasured five times. The intraclass correlation was 0.98 (0.93, 0.99), indicating a reliable measure.

\section{Variance components analysis of light-dark hair color}

Variance components analysis was performed to determine the proportion of variation in the light-dark continuum of hair color (A650t) that is caused by genetic and environmental influences. These analyses were based on the most parsimonious model to explain means and variances in A650t index (transformed A650 index) across birth order, zygosity and sex 
a)

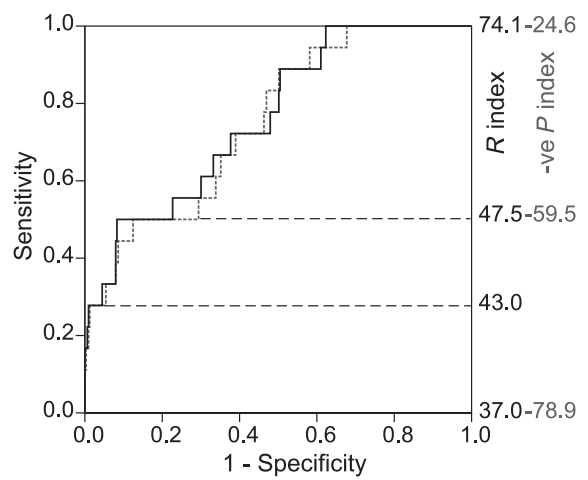

b)

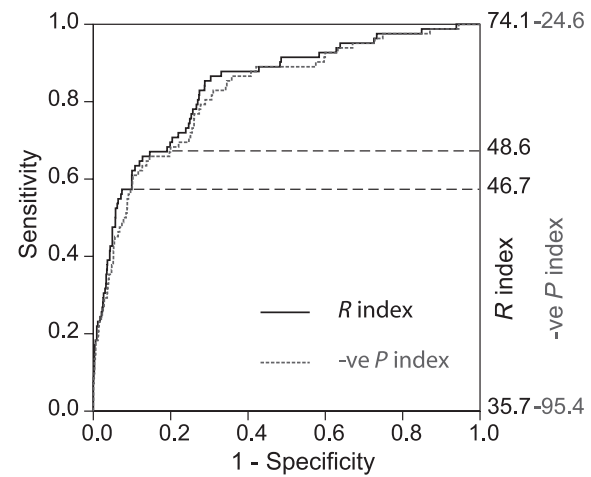

Figure 5. The receiver operating characteristic curves to determine the sensitivity of the $R$ and $P$ indices to distinguish (a) MC1R homozygotes at highly penetrant red hair alleles (R) from MC1R wildtype homozygotes and (b) red headed individuals from those of other colors. Note: The scale on the right-hand side of the charts is dependant upon the distribution of the $R$ index. Number of individuals is described in Figs. 3 and 4 .

(21). Starting with a model where all possible mean and variance differences were modeled, stepwise simplifications of the model were tested using the likelihood ratio test. Females had, on average, lighter hair, on the A650t scale, than males.

The correlation in A650t index scores between monozygotic twin pairs was greater than the correlation in scores between dizygotic twin pairs $(P<0.00001$; Table 3$)$. This confirms that familial aggregation in light-dark hair color is, at least partly, due to genetic influences. The correlation within brother-sister twin pairs was significantly lower than the correlation within brother-brother and sister-sister dizygotic twin pairs $(P \approx 0.01)$. This suggests that there may be qualitative differences in the genetic influences on the A650t index between sexes. This conclusion could not be drawn by simply rating hair color.

The dizygotic twin pair correlations were greater than the expected halving of the monozygotic twin pair correlations for the same sex zygosity groups (ff $r=0.77$ vs $r=0.63 ; \mathrm{mm}$ $r=0.76$ vs $r=0.45$ ). This suggests that common environmental influences were a greater source of variation than nonadditive genetic influences for the A650t index. To account for the qualitative difference in genetic influence between sexes, a general sex-limited ACE model was fitted (Table 4). In addition to allowing for different proportions of variation influencing the A650t index between sexes, this model also

Table 3. Maximum likelihood estimates of twin pair Pearson correlations and $95 \%$ confidence intervals for the A650t and $R$ indices by zygosity group. Polychoric correlations are presented for ordered hair color.

\begin{tabular}{|c|c|c|c|c|c|c|c|c|}
\hline & \multicolumn{3}{|c|}{ A650t index } & \multicolumn{2}{|c|}{ Ordered ratings } & \multicolumn{3}{|c|}{$R$ index* } \\
\hline & $n$ & $r$ & $95 \% \mathrm{CI}$ & $r$ & $95 \% \mathrm{CI}$ & $n$ & $r$ & $95 \% \mathrm{CI}$ \\
\hline mzff & 137 & 0.77 & $0.70-0.82$ & 0.999 & $0.997-0.999$ & 276 & 0.57 & $0.49-0.63$ \\
\hline mzmm & 127 & 0.76 & $0.69-0.82$ & & & & & \\
\hline dzff & 113 & 0.63 & $0.50-0.72$ & 0.59 & $0.46-0.70$ & & & \\
\hline dzmm & 115 & 0.45 & $0.30-0.57$ & & & 443 & 0.23 & $0.19-0.32$ \\
\hline dzos & 176 & 0.35 & $0.22-0.44$ & 0.45 & $0.29-0.59$ & & & \\
\hline
\end{tabular}

*There was no significant difference in twin pair correlations between the same sex identical twins and between the same sex and opposite sex nonidentical twins. The correlations are pooled within zygosity. includes a male-specific additive genetic influence. The proportion of variation resulting from familial aggregation was similar in both sexes $(\approx 0.77)$. Additive genetic influences explain $55 \%$ and $58 \%$ of variation in the A650t index within females and males, respectively. The additive genetic influence on the A650t index in males was, predominantly, qualitatively different from those that influence the index in females. Some $20 \%$ of variation in the A650t index is due to environmental influences common within twin pairs.

A genetic analysis of ordered ratings of hair color in this study shows that additive genetic influences explain $92 \%(88$, $99.9 \%$ ) of variation in hair color. The majority of the residual is due to environmental influences common within twin pairs $(8 \%[4,17 \%])$. This result is similar to that in a study by Matheny and Dolan (29) of 390 twin pairs rated for hair color using swatches. They showed that $70 \%$ of variation at age 6 was due to additive genetic influences and $25 \%$ was due to environmental influences common between twin pairs, across both sexes.

\section{Variance components analysis of the $R$ index}

Testing of hypotheses regarding homogeneity of means and variances was done prior to variance components analysis. Females had, on average, redder hair $(P<0.00001)$ and greater variation in $R$ index scores $(P \approx 0.001)$ than males. Again, the correlation between monozygotic twin pairs was greater than the correlation between dizygotic twin pairs $(P<0.00001)$. Dizygotic twin pair covariances were less than half the monozygotic twin pair covariances, suggesting that nonadditive genetic influences were a more important source of variation than common environmental genetic influences. A model was fitted where the ADE components of variation influencing males could be multiplied by a scalar multiple to determine the ADE components influencing variation in $R$ index values in females. The parameter explaining the scalar difference between sexes could not be removed without a significant difference in fit (Table 5). Nonadditive genetic influences, although not significant, explained $31 \%$ of variation in the $R$ index (Table 5). This may be due to MC1R variants. Cumulatively, additive and nonadditive genetic influences explain $58 \%$ of variation in the $R$ index with the 
Table 4. Maximum likelihood estimation and fit of genetic models for the A650t index with standardized estimates of additive genetic (A), common environmental (C), and unique environmental (E) influences and their $95 \%$ confidence intervals.

\begin{tabular}{lccccccccc}
\hline & $\begin{array}{c}-2 \log \\
\text { likelihood }\end{array}$ & d.f. & $\mathrm{A}_{\text {males only }}$ & $\mathrm{C}_{\text {males }}$ & $\mathrm{E}_{\text {males }}$ & $\mathrm{A}_{\text {males }}$ & $\mathrm{A}_{\text {females }}$ & $\mathrm{C}_{\text {females }}$ & $\mathrm{E}_{\text {females }}$ \\
\hline $\begin{array}{l}\text { I. General } \\
\text { sex-limited }\end{array}$ & 2436.00 & 1594 & 0.45 & 0.19 & 0.23 & 0.13 & 0.55 & 0.22 & 0.23 \\
$(0.00-0.62)$ & $(0.00-0.39)$ & $(0.18-0.30)$ & $(0.00-0.80)$ & $(0.34-0.79)$ & $(0.00-0.41)$ & $(0.18-0.29)$
\end{tabular}

Table 5. Maximum likelihood estimation and fit of genetic models for the $R$ index with standardized estimates of additive genetic (A), nonadditive genetic (D), and unique environmental (E) influences and their $95 \%$ confidence intervals.

\begin{tabular}{|c|c|c|c|c|c|c|c|c|c|c|}
\hline & $\begin{array}{c}-2 \log \\
\text { likelihood }\end{array}$ & d.f. & $v s$ model & $\Delta \chi^{2}$ & $\Delta$ d.f. & $P$ & A & $\mathrm{D}$ & E & $\begin{array}{l}\text { Scalar } k \\
\text { (males)* }^{*}\end{array}$ \\
\hline $\begin{array}{l}\text { I. Scalar sex-limited } \\
\text { ADE model }\end{array}$ & 10407.93 & 1683 & & & & & $\begin{array}{c}0.27 \\
(0.00-0.54)\end{array}$ & $\begin{array}{c}0.31 \\
(0.01-0.61)\end{array}$ & $\begin{array}{c}0.42 \\
(0.36-0.50)\end{array}$ & 0.86 \\
\hline II. ADE model & 10426.00 & 1684 & I & 18.08 & 1 & $<0.0001$ & $\begin{array}{c}0.27 \\
(0.00-0.54)\end{array}$ & $\begin{array}{c}0.30 \\
(0.01-0.61)\end{array}$ & $\begin{array}{c}0.43 \\
(0.36-0.51)\end{array}$ & - \\
\hline III. AE model & 10412.11 & 1684 & I & 4.19 & 1 & 0.41 & $\begin{array}{c}0.54 \\
(0.46-0.61)\end{array}$ & - & $\begin{array}{c}0.46 \\
(0.39-0.54)\end{array}$ & 0.86 \\
\hline
\end{tabular}

*The variance components of females are multiplied by this scalar constant to determine the variance components for males. The values suggest males have less variation than females.

residual variation due to environmental influences unique between twins.

\section{DISCUSSION}

This investigation sought to determine the spectrophotometric approximations of pheomelanin and eumelanin that best explain observed hair color. Regardless of color classification, hairs absorbed light in the blue-violet portion of the spectrum (below $450 \mathrm{~nm}$ ). Spectrophotometrically, the continuum of light-dark hair color is due to hair absorbing light in the green through red portion of the spectrum. If one sample absorbs a greater proportion of light in the green portion of the spectrum than another, then the former hair sample is expected to appear more "red" than the latter. It is known that red hair contains a greater proportion of pheomelanin than brown/ black hair colors (3). So red hair, and possibly pheomelanin, is best quantified, as Reed (14) suggested, by calculating "the ratio of reflectance at 530 to that at 650 ." It is highly probable that pheomelanin absorbs light in the green portion of the spectrum. Although red hair absorbs some light in the red portion of the spectrum, variation in absorbance around $650 \mathrm{~nm}$ appears to have less influence in distinguishing red hair from other colors.

Although these results suggest that eumelanin absorbs light across the red and green portion of the spectrum, it is confounded by pheomelanin content in the green portion of the spectrum. Therefore, it is expected that eumelanin quantity is best approximated in the red portion of the spectrum. In this study, absorbance at $650 \mathrm{~nm}$ and reflectance at $600 \mathrm{~nm}$ were the measures that were best able to distinguish between hair colors on the light-dark spectrum. Once transformed, absorbance at $650 \mathrm{~nm}$ explained ordered ratings of hair color similar to the melanin index. This may follow from their being transformations of reflectance at or around $650 \mathrm{~nm}$.

Individuals with predominantly pheomelanin will also absorb light in the red portion of the spectrum, confounding the measure of eumelanin. Although it is suggested that individuals with red hair be removed from any spectrophotometric analysis of eumelanin, the $R$ index is not able to fully distinguish those with red hair from those of other hair colors. Although most individuals below an $R$ index value of 39.9 have red hair, over $80 \%$ of individuals classified as having red hair have $R$ index values greater than 39.9, overlapping with the distributions of the $R$ indices for other hair colors. Although Reed (14) does note that many red headed males have $R$ index values below 48 and many red headed females have $R$ index values below 45, he concluded that there is no point on the distribution of the $R$ index that can be used to distinguish between red and non-RHC. That conclusion applies to this study of the $R$ index as well.

Overall, these results concord with those of Ozeki et al. (11) that absorbance at $500 \mathrm{~nm}$ explains the total amount of melanin and the suggestion by Ozeki et al. (12) that absorbance at $650 \mathrm{~nm}$ is proportional to eumelanin quantity in human hair. The results also confirm the melanin index as a measure of light-dark hair color. Although the CIE Lab index was not investigated here, the $a^{*}$ dimension of this index may be of value in determining pheomelanin content in hair as it is a continuum of red-green. The measures discussed here employ wavelengths in the visible portion of the spectrum. This allows both eumelanin and pheomelanin quantity to be approximated rapidly using one instrument. In addition, the measures are not confounded by the major structural component of hair, keratin, that absorbs light in the UVB portion of the spectrum (30). So, the $R$ index and absorbance at $650 \mathrm{~nm}$ are orthogonal indices that capture the two perceived dimensions of hair color, red and light-dark, respectively.

There is also considerable overlap in the distributions of the $R$ index between those homozygous for the wildtype MC1R alleles and those homozygous for the high penetrance $\mathrm{R}$ MC1R alleles. Assuming that the $R$ index captures variation in red hair, this confirms that MC1R does not altogether explain variation in red hair. Variation in the $R$ index was due to both 
additive and nonadditive genetic influences. We expect that the effect of the nonadditive genetic influences to be due to MC1R variant alleles within the population.

Continuous, objective measures have greater power to detect the effect of variance components contributing to variation than subjective, categorized ratings (31). Variance components analysis of light-dark hair color identified some qualitative differences in the sources of additive genetic influence between sexes. Interestingly, this result is consistent with the literature on gender differences in evolutionary biology. A study by Thelen (32) suggests that eumelanin in females is under sexual selection for the rarest color in a population while hair color in males undergoes natural selection for the most unobtrusive color (this area is reviewed by Frost) (33). Subjective ratings of hair color were not able to detect qualitative or quantitative differences in the sources of variation between sexes.

The study by Matheny and Dolan (29) using the Haarfarbentafel rating of hair color, like our ordered rating of hair color, did not suggest any difference in the sources of variation between sexes. With regard to study design, although there is a significant increase in time and resources needed to produce spectrophotometric curves compared to rating color by observation, there is greater power to detect the influences that cause variation in hair color. Testing for model parsimony, including sex differences, is particularly important if subsequent analyses and tests will be based on that model. Although these results show A650t to be a surrogate for eumelanin after removing individuals with red hair, there may be a number of confounding factors such as light scatter and melanin particle size. For example, Liu et al. (34) showed that melanosomes from black hairs absorb 2.9 times as much light at $500 \mathrm{~nm}$ as melanosomes from red hairs. Although we expect such factors to have a small effect on variation, biochemical analysis or electron paramagnetic resonance spectroscopy would provide a more accurate measure, although at greater cost (35).

Acknowledgements - This work was supported by NHMRC grants 950998, 981339, 241944 (to N.G.M.) and NIH grant CA88363 (to Dr. Nick Hayward). We would like to thank Ann Eldridge and Marlene Grace for sample collection; the team at DNAPrint Genomics for generating the spectrophotometric curves; Anjali Henders, Megan Campbell and the staff of the Molecular Epidemiology Laboratory for blood processing and DNA extraction; and Gu Zhu, Sarah Medland and Allan McRae for their assistance with data analysis and useful comments. We would also like to thank the twins as well as their parents and siblings for their participation.

\section{REFERENCES}

1. Harrison, G. A. (1973) Differences in human pigmentation: Measurement, geographic variation, and causes. J. Invest. Dermatol. 60, 418-426.

2. Wakamatsu, K. and S. Ito (2002) Advanced chemical methods in melanin determination. Pigment Cell Res. 15, 174-183.

3. Borges, C. R., J. C. Roberts, D. G. Wilkins and D. E. Rollins (2001) Relationship of melanin degradation products to actual melanin content: Application to human hair. Anal. Biochem. 290, $116-125$.

4. Sturm, R. A. (2006) A golden age of human pigmentation genetics. Trends Genet. 22, 464-468.

5. Hunt, G., S. Kyne, K. Wakamatsu, S. Ito and A. J. Thody (1995) Nle4DPhe7 alpha-melanocyte-stimulating hormone increases the eumelanin:phaeomelanin ratio in cultured human melanocytes. $J$. Invest. Dermatol. 104, 83-85.
6. Valverde, P., E. Healy, I. Jackson, J. L. Rees and A. J. Thody (1995) Variants of the melanocyte-stimulating hormone receptor gene are associated with red hair and fair skin in humans. Nat. Genet. 11, 328-330.

7. Sturm, R. A., D. L. Duffy, N. F. Box, W. Chen, D. J. Smit, D. L. Brown, J. L. Stow, J. H. Leonard and N. G. Martin (2003) The role of melanocortin-1 receptor polymorphism in skin cancer risk phenotypes. Pigment Cell Res. 16, 266-272.

8. Box, N. F., J. R. Wyeth, L. E. O'Gorman, N. G. Martin and R. A. Sturm (1997) Characterization of melanocyte stimulating hormone receptor variant alleles in twins with red hair. Hum. Mol. Genet. 6, 1891-1897.

9. Harrison, G. A. and J. J. Owen (1964) Studies on the inheritance of human skin colour. Ann. Hum. Genet. 28, 27-37.

10. Edwards, E. A. and S. Q. Duntley (1939) The pigments and colour of human living skin. Am. J. Anat. 65, 1

11. Ozeki, H., S. Ito, K. Wakamatsu and T. Hirobe (1995) Chemical characterization of hair melanins in various coat-color mutants of mice. J. Invest. Dermatol. 105, 361-366.

12. Ozeki, H., S. Ito, K. Wakamatsu and A. J. Thody (1996) Spectrophotometric characterization of eumelanin and pheomelanin in hair. Pigment Cell Res. 9, 265-270.

13. Gardner, B. B. and D. L. MacAdam (1934) Colorimetric analysis of hair color. Am. J. Phys. Anthropol. 19, 187-201.

14. Reed, T. E. (1952) Red hair colour as a genetical character. Ann. Eugen. 17, 115-139.

15. Little, M. A. and M. E. Wolff (1981) Skin and hair reflectance in women with red hair. Ann. Hum. Biol. 8, 231-241.

16. McGregor, B., J. Pfitzner, G. Zhu, M. Grace, A. Eldridge, J. Pearson, C. Mayne, J. F. Aitken, A. C. Green and N. G. Martin (1999) Genetic and environmental contributions to size, color, shape, and other characteristics of melanocytic naevi in a sample of adolescent twins. Genet. Epidemiol. 16, 40-53.

17. Zhu, G., D. L. Duffy, A. Eldridge, M. Grace, C. Mayne, L. O’Gorman, J. F. Aitken, M. C. Neale, N. K. Hayward, A. C. Green and N. G. Martin (1999) A major quantitative-trait locus for mole density is linked to the familial melanoma gene CDKN2A: A maximum-likelihood combined linkage and association analysis in twins and their sibs. Am. J. Hum. Genet. 65, 483492.

18. Miller, S. A., D. D. Dykes and H. F. Polesky (1988) A simple salting out procedure for extracting DNA from human nucleated cells. Nucleic Acids Res. 16, 1215.

19. Duffy, D. L., N. F. Box, W. Chen, J. S. Palmer, G. W. Montgomery, M. R. James, N. K. Hayward, N. G. Martin and R. A. Sturm (2004) Interactive effects of MC1R and OCA2 on melanoma risk phenotypes. Hum. Mol. Genet. 13, 447-461.

20. Duffy, D. L., G. W. Montgomery, W. Chen, Z. Z. Zhao, L. Le, M. R. James, N. K. Hayward, N. G. Martin and R. A. Sturm (2007) A three-single-nucleotide polymorphism haplotype in intron 1 of OCA2 explains most human eye-color variation. Am. J. Hum. Genet. 80, 241-252.

21. Evans, D. M., I. H. Frazer and N. G. Martin (1999) Genetic and environmental causes of variation in basal levels of blood cells. Twin Res. 2, 250-257.

22. Shekar, S. N., M. Luciano, D. L. Duffy and N. G. Martin (2005) Genetic and environmental influences on skin pattern deterioration. J. Invest. Dermatol. 125, 1119-1129.

23. Neale, M. C. and L. R. Cardon (1992) Methodology for Genetic Studies of Twins and Families. Kluwer Academic Publishers, Dordrecht, Boston, xxv, 496 pp.

24. Eaves, L. (1977) Inferring the causes of human variation. $J . R$. Stat. Soc. Ser. A-G. 140, 324-355.

25. Posthuma, D., A. L. Beem, E. J. de Geus, G. C. van Baal, J. B. von Hjelmborg, I. Iachine and D. I. Boomsma (2003) Theory and practice in quantitative genetics. Twin Res. 6, 361-376.

26. Neale, M. C., S. M. Boker, G. Xie and H. H. Maes (2002) Mx: Statistical Modelling. Department of Psychiatry, Richmond, VA.

27. Medland, S. E. (2004) Alternate parameterization for scalar and non-scalar sex-limitation models in Mx. Twin Res. 7, 299-305.

28. Shriver, M. D. and E. J. Parra (2000) Comparison of narrow-band reflectance spectroscopy and tristimulus colorimetry for measurements of skin and hair color in persons of different biological ancestry. Am. J. Phys. Anthropol. 112, 17-27. 
29. Matheny, A. P., Jr and A. B. Dolan (1975) Sex and genetic differences in hair color changes during early childhood. Am. J. Phys. Anthropol. 42, 53-56.

30. Nogueira, A. C., L. E. Dicelio and I. Joekes (2006) About photodamage of human hair. Photochem. Photobiol. Sci. 5, 165-169.

31. Neale, M. C., L. J. Eaves and K. S. Kendler (1994) The power of the classical twin study to resolve variation in threshold traits. Behav. Genet. 24, 239-258.

32. Thelen, T. H. (1983) Minority type human mate preference. Soc. Biol. 30, 162-180.
33. Frost, P. (2006) European hair and eye color. A case of frequency-dependent sexual selection? Evol. Hum. Behav. 27, $85-103$.

34. Liu, Y., L. Hong, K. Wakamatsu, S. Ito, B. Adhyaru, C. Y. Cheng, C. R. Bowers and J. D. Simon (2005) Comparison of structural and chemical properties of black and red human hair melanosomes. Photochem. Photobiol. 81, 135-144.

35. Ito, S., K. Wakamatsu and H. Ozeki (2000) Chemical analysis of melanins and its application to the study of the regulation of melanogenesis. Pigment Cell Res. 13(Suppl. 8), 103-109. 